\title{
Is the whole greater than the sum of its parts? De novo assembly strategies for bacterial genomes based on paired-end sequencing
}

\author{
Ting-Wen Chen ${ }^{1}$, Ruei-Chi Gan ${ }^{1}$, Yi-Feng Chang ${ }^{1,2}$, Wei-Chao Liao ${ }^{1}$, Timothy H. Wu³ , Chi-Ching Lee ${ }^{1}$, \\ Po-Jung Huang ${ }^{1}$, Cheng-Yang Lee', Yi-Ywan M. Chen ${ }^{4,5}$, Cheng-Hsun Chiu ${ }^{6}$ and Petrus Tang ${ }^{1,5,6^{*}}$
}

\begin{abstract}
Background: Whole genome sequence construction is becoming increasingly feasible because of advances in next generation sequencing (NGS), including increasing throughput and read length. By simply overlapping paired-end reads, we can obtain longer reads with higher accuracy, which can facilitate the assembly process. However, the influences of different library sizes and assembly methods on paired-end sequencing-based de novo assembly remain poorly understood.
\end{abstract}

Results: We used 250 bp Illumina Miseq paired-end reads of different library sizes generated from genomic DNA from Escherichia coli DH1 and Streptococcus parasanguinis FW213 to compare the assembly results of different library sizes and assembly approaches. Our data indicate that overlapping paired-end reads can increase read accuracy but sometimes cause insertion or deletions. Regarding genome assembly, merged reads only outcompete original paired-end reads when coverage depth is low, and larger libraries tend to yield better assembly results. These results imply that distance information is the most critical factor during assembly. Our results also indicate that when depth is sufficiently high, assembly from subsets can sometimes produce better results.

Conclusions: In summary, this study provides systematic evaluations of de novo assembly from paired end sequencing data. Among the assembly strategies, we find that overlapping paired-end reads is not always beneficial for bacteria genome assembly and should be avoided or used with caution especially for genomes containing high fraction of repetitive sequences. Because increasing numbers of projects aim at bacteria genome sequencing, our study provides valuable suggestions for the field of genomic sequence construction.

\section{Background}

Although many assemblers have been proposed for de novo assembly with NGS data, the inherent shorter length and higher error rate of short read NGS data still hinder the reconstruction of bacterial genomes. Although traditional Sanger sequencing obtained reads up to 1000 base pairs long with accuracy as high as $99.999 \%$, NGS generates high throughput sequences with reduced read lengths and qualities [1]. Read length is undoubtedly critical in de novo assembly. The most

\footnotetext{
* Correspondence: petang@mail.cgu.edu.tw

'Bioinformatics Core Laboratory, Molecular Medicine Research Center, Chang Gung University, Taoyuan, Taiwan

${ }^{5}$ Graduate Institute of Biomedical Sciences, Chang Gung University, Taoyuan, Taiwan

Full list of author information is available at the end of the article
}

extreme example is a read as long as the entire genome that could complete construction of the entire genome. Third generation single-molecule sequencing has successfully generated reads longer than $10-15 \mathrm{~kb}$ or even approaching $150 \mathrm{~kb}$ for PacBio and MinION sequencers, respectively [2-4]. However, PacBio and MinION generate reads with sequencing errors rates as high as 15 and $35 \%$ respectively $[4,5]$. Several methods are proposed to deal with such high error rates [4-6]. Recently Illumina TruSeq Synthetic Long-Reads (previously known as Moleculo) is also proposed to provide longer reads [7]. Nevertheless, currently what we usually have reads as short as several hundreds of bps long that require sophisticated algorithms or strategies for de novo assembly. Many de novo assemblers utilizing the de Bruijn graphbased approach were developed for NGS reads, such as 
Velvet, SOAPdenovo, ALLPATHS, Edena, EULER-SR, and EULER-USR [8-14]. These methods construct de Bruijn graphs from short reads and obtain contigs by solving the graphs. Other studies come from a theoretical perspective and discuss whether these short reads are intrinsically sufficient for genome assembly $[15,16]$. Whiteford et al. demonstrated that 90 to $97 \%$ of the $E$. coli genome could be reconstructed with reads as short as $50 \mathrm{bp}$. However, because of repeats in the E. coli genome, it required read lengths of up to $475 \mathrm{bp}$ to allow $99 \%$ of the reads to uniquely map to the genome [16]. The existence of repeat sequences largely increased the complexity for resolving the whole genome sequence. The problem was only partially solved when the de Bruijn graph-based approach was proposed, and only reads longer than the repeat sequence or structural information provided by mate pair sequencing can possibly overcome this difficulty $[15,17]$. In addition to reduced length, higher error rates in NGS reads also contribute to the difficulty of genome reconstruction $[18,19]$. Assemblers based on the de Bruijn approach may be largely complicated by sequencing errors. Several studies have focused on providing sequencing error correction tools that can largely improve assembly results $[20,21]$.

Several studies have assembled whole genome sequences by combining data from both Illumina and 454 sequencing technologies that can compensate one another's weaknesses $[10,13]$. Long reads from 454 can assist short reads produced by Illumina in resolving repeat regions. As the technology rapidly advances, read length is becoming longer, and longer reads are undoubtedly helpful in the de novo assembly of genomes. Currently, the read length of the Illumina MiSeq has reached as long as $300 \mathrm{bp}$, although it remains shorter than the Roche 454 sequencing reads. Nevertheless, we can easily generate longer reads by overlapping Illumina pairedend reads. Several tools have already been developed for merging paired-end reads, such as the merge module in CLC, PEAR, FLASH, PANDAseq, Stitch and FastqJoin [22-27]. Paired-end read merging allows us to generate reads lengths comparable to 454 . However, how these merged reads influence assembly results remains unanswered. In this study, we used real sequencing data to demonstrate the potential effects of merged reads in real world genome assembly.

To investigate the effect of merged paired-end reads in de novo assembly, we explored the sequence accuracy of merged reads and tried different assembly strategies with the same Illumina datasets. We generated Illumina paired-end reads with four different library sizes (300 bp, $400 \mathrm{bp}, 500 \mathrm{bp}$ and $600 \mathrm{bp}$ ) from the genomes of Escherichia coli DH1 and Streptococcus parasanguinis FW213. These genomes were chosen as representative bacterial genomes because they have considerably different GC contents and genome complexities, both properties known to influence sequencing quality and assembly processes. E. coli DH1 and S. parasanguinis FW213 have intermediate (50.8 \%) and low (41.7 \%) GC contents, respectively. S. parasanguinis FW213 has a higher proportion of short tandem repeats and long repeats in its genome compared with E. coli DH1. We explored the accuracy of these reads and the de novo assembly results from these reads. Although MiSeq has been shown to provide the lowest error rate compared with the 454 GS Junior and Ion Torrent PGM, which both produce homopolymer-associated indel (insertion/deletion) error [28], we still observed certain levels of sequencing errors, even after reads were trimmed. We also observed that these errors could be significantly reduced by simply merging paired-end reads. However, the merge step sometimes also largely increased indel errors, particularly for reads from libraries with few overlapping regions or from genomes with many tandem repeats. Given that the merged reads had higher sequence qualities and longer lengths, we further performed reconstruction of the genome sequences using different de novo assembly strategies with either paired-end reads or merged reads for different library sizes. In addition to assembly strategies, information on how many reads should be used will be helpful in experimental design. Several studies already provide suggestions on the optimum depth for genome assembly $[29,30]$. By examining the assembly results generated from real data, we discuss how paired-end reads should be used to generate the best assembly results. Our study provides invaluable and useful suggestions for hundreds of ongoing bacterial genome reconstruction projects.

\section{Methods}

\section{Sequence read preparation}

Total cellular DNA from E. coli DH1 and S. parasanguinis FW213 were prepared by standard protocols [31]. Four paired-end libraries containing different sizes of DNA fragments (300 bp, $400 \mathrm{bp}, 500 \mathrm{bp}$ and $600 \mathrm{bp}$ ) were prepared by Ovation Ultralow Library Systems (NuGEN) for both $E$. coli DH1 and S. parasanguinis FW213. The libraries were sequenced by an Illumina MiSeq sequencer. A total of eight different datasets of paired-end 2 X $250 \mathrm{bp}$ reads were generated (SRP060735). For sequence quality estimation, calculate_stats from seq_crumbs was used to estimate the read qualities for each dataset [32]. For further de novo assembly analysis, reads were trimmed to fit the quality threshold of Phred quality scores larger than Q20 and lengths larger than $50 \mathrm{bp}$.

\section{Mapping reads to reference genomes}

The reference genome sequences of $E$. coli DH1 (NC_017625) and S. parasanguinis FW213 (NC_017905) 
were downloaded from NCBI [33]. We used seqtk [34] to randomly select $40 \%$ of all original raw reads 5 times. These subsets of raw reads were merged with or without trimming. The resulting dataset of reads (raw reads, trimmed reads and merged reads) were all mapped back to their corresponding reference genomes with BWAMEM using default parameters [35]. Mismatch and mapping rates were calculated from the output mapped bam files using sam-stats from ea-utils [24].

\section{Overlapping paired reads}

To investigate the effect of overlapping reads, the "merge overlapping pairs" module in the CLC Genomics workbench was used to overlap the paired-end reads [26]. For reads that could be overlapped together (referred to as "Group M" henceforth), their overlapping reads and original paired-end reads were both generated as separate files using several in-house scripts.

\section{de novo assembly}

The CLC Genomics Workbench [26] was used for de novo assembly with default parameters. There were five different assembly strategies. The original raw reads were assembled as paired-end reads (Group A [PE]) or as singleend reads (Group A [SE]). Reads that overlapped were either assembled as paired-end reads (Group M [PE]) or as single-end reads (Group M [SE]) after overlapping. All of these merged single-end reads and the rest of the nonoverlapped paired-end reads were also assembled together (Group A $[\mathrm{PE}+\mathrm{SE}]$ ). For each assembly strategy, an inhouse, multithreaded Perl script was used to randomly generate different numbers of reads from the total number of reads. These reads were randomly sampled from the raw data, and 9260/4340 paired reads were treated as coverage depths of $1 \mathrm{X}$ for E. coli DH1 and S. parasanguinis FW213 which have genome sizes of $4.63 \mathrm{Mb}$ and $2.17 \mathrm{Mb}$, respectively. We randomly selected three independent datasets from each depth to estimate variance.

\section{Repeat region analysis}

The Tandem Repeats Finder was used to identify tandem repeats in both the E. coli DH1and S. parasanguinis FW213 [36] genomes. Only tandem repeats with matches larger than $80 \%$ were included and analyzed. For short tandem repeat analysis, tandem repeats with sizes smaller than $100 \mathrm{bp}$ were included. These tandem repeat period sizes were multiplied by their corresponding copy numbers and then summed to estimate the percent of the genome that was located within tandem repeats.

\section{Estimating assembly quality}

All contigs generated from de novo assembly were further evaluated by QUAST [37]. From the QUAST report, several important and representative statistical values, such as N50, genome fraction (\%), number of misassemblies, number of genes covered, and number of contigs and number of indels per $100 \mathrm{~kb}$ were used to evaluate the assembly qualities.

\section{Results and discussion}

Merging paired-end reads improves their accuracy

To investigate the effect of overlapping paired-end reads on the accuracy of merged reads, we generated eight different datasets from the E. coli DH1 and S. parasanguinis FW213 genomes. We produced 2 X 250 paired-end reads from each genome. We designed four different library sizes: $300 \mathrm{bp}, 400 \mathrm{bp}, 500 \mathrm{bp}$ and $600 \mathrm{bp}$ for each genome, and all reads were generated by the MiSeq platform. All eight datasets contained more than 1 million reads and high sequence quality, with average Phred scores greater than 30 (Table 1). The CLC workbench was then used to merge overlapped paired reads. This merging step looked for overlapping regions from the ends of paired reads and merged these overlapped regions. If any conflicts in the overlapped region existed, the read with the higher quality score was used. We defined two read datasets from this merging step: Group A that included all reads and Group M that included only a subset of reads that could be merged. As expected, most of the reads from the library size of 300 could be merged together, and the percentage of read pairs that could be merged decreased as the library size increased (Table 1).

The influence of sequence accuracy after the merging step was the first issue we investigated. Because base quality usually drops towards the end of the read, some sequencing errors can be corrected during the merging step. To survey the extent and effect of correcting sequencing errors in this manner, we mapped raw paired reads and merged reads from Group $M$ to the reference genomes. We observed that the mismatch rate for Group M was significantly lower than that for Group A (Fig. 1). This result demonstrates that only raw reads with higher accuracy could be merged. Although the merged reads did not extensively increase the mapping rate, they did significantly decrease the mismatch rate. Although the merging step only involved partial read regions, this step corrected 10.2 to $67.3 \%$ of the mismatches in raw reads (Additional file 1: Table S1). The accuracy improvement was most significant in the smallest library that had the longest overlapping regions on average (Fig. 1a). We further examined whether the same sequence accuracy improvement could be achieved by sequence trimming, which is one of the most commonly used strategies for eliminating low quality bases. Raw reads were trimmed for quality (Phred score larger than Q20) and length (longer than $50 \mathrm{bp}$ ) and then mapped back to the reference genomes with and without the merging step. We still observed a significant decrease in 
Table 1 Summary of paired-end reads used in this study

\begin{tabular}{|c|c|c|c|c|c|c|}
\hline Reference genome & Library size & Read number & Average quality $^{\mathrm{a}}$ & $\mathrm{Q} 20^{\mathrm{b}}$ & $\mathrm{Q} 30^{\mathrm{b}}$ & Group $M^{c}$ \\
\hline E. coli DH1 & $300 \mathrm{bp}$ & $1,894,068$ & 34.31 & $90.15 \%$ & $85.27 \%$ & $71.59 \%$ \\
\hline E. coli DH1 & $400 \mathrm{bp}$ & $2,221,312$ & 33.91 & $88.94 \%$ & $83.61 \%$ & $69.21 \%$ \\
\hline E. coli DH1 & $500 \mathrm{bp}$ & $2,093,116$ & 33.50 & $87.63 \%$ & $81.87 \%$ & $25.12 \%$ \\
\hline E. coli DH1 & $600 \mathrm{bp}$ & $1,548,836$ & 32.79 & $85.29 \%$ & $78.78 \%$ & $0.85 \%$ \\
\hline S. Parasanguinis FW213 & $300 \mathrm{bp}$ & $2,162,006$ & 35.84 & $93.47 \%$ & $90.77 \%$ & $82.05 \%$ \\
\hline S. Parasanguinis FW213 & $400 \mathrm{bp}$ & $1,968,178$ & 35.60 & $92.67 \%$ & $89.76 \%$ & $81.04 \%$ \\
\hline S. Parasanguinis FW213 & $500 \mathrm{bp}$ & $1,886,084$ & 35.21 & $91.55 \%$ & $88.26 \%$ & $19.03 \%$ \\
\hline S. Parasanguinis FW213 & $600 \mathrm{bp}$ & $1,745,876$ & 34.61 & $89.58 \%$ & $85.67 \%$ & $6.82 \%$ \\
\hline
\end{tabular}

${ }^{\mathrm{b}}$ The percentage of bases in the reads with a Phred score equal or larger than 20 or 30

'Percentage of paired reads that can be merged

mismatch rate for trimmed paired-end reads from library sizes of $300 \mathrm{bp}$ and $400 \mathrm{bp}$ but an opposite trend for reads from larger libraries (Fig. 1b). Similar decrease and increase pattern in mismatch rates were found when reads were merged by FLASH, PANDAseq or PEAR (Additional file 1: Table S2). This increase in mismatch rate may be random because fewer reads belonged to Group $M$ for large library size. In our example, the number of reads from the 600 bp library of Group M was approximately $1 /$ 100 (E. coli DH1) and 1/10 (S. parasanguinis FW213) the number of reads from other library sizes. This small size in reads number make this group more susceptible to sampling fluctuation. However, the merge step may introduce incorrect sequences by erroneously merging nonoverlapping region. Because these mistaken merges were most likely to occur in regions with repeats, we further explored the insertion and deletion rates from previous mapping results (Fig. 2). We observed that reads from Group A had similar insertion and deletion rates whether mapped as paired-end or single-end reads. However, both the insertion and deletion rates significantly increased for merged reads from Group M with library sizes of $500 \mathrm{bp}$ and $600 \mathrm{bp}$. Increase in both insertion and deletion rates were also found when reads were merged by FLASH, PANDAseq or PEAR (Additional file 1: Table S3 and Table S4). This result implies that when the overlapped regions are short or non-existent, the merging step may cause a significant number of indel errors, explaining the increase of the mismatch rate observed for the merged reads from large library sizes.

\section{Location information from paired-end reads largely promotes de novo assembly}

Based on the improved accuracy observed with the overlapped paired-end reads, we were interested in investigating how the improvement influences the process of de novo assembly. For all library sizes, we assembled paired raw reads as paired-end reads (Group A [PE]) or as single-end reads (Group A [SE]). We also assembled the merged reads together with the non-overlapping reads (Group A $[\mathrm{PE}+\mathrm{SE}]$ ). To compare the assembly results for different library sizes, we used N50 (N50 is the length for which the collection of all contigs of that length or longer contains at least half of the sum of the lengths of all contigs) to represent the integrity of assembly result [38]. As shown in Fig. 3, as read number increased, N50 increased and then plateaued. Within all assembly strategies, Group A [PE] always yielded the highest N50 for all library sizes. Group A [PE + SE] had similar performance as Group A [PE] in E. coli DH1 but did not generate a higher N50 value compared to Group A [PE]. Nevertheless, Group A [PE + SE] had significant drops of N50 were observed for library sizes of $300 \mathrm{bp}$ and $400 \mathrm{bp}$ in S. parasanguinis FW213 (Fig. 3f). This drop also existed in large libraries (500 bp or $600 \mathrm{bp}$ ) that had much lower ratios of overlapping reads.

Larger library size typically yielded a higher N50 value given the same read number and assembly strategy (Fig. 3a and b). We also examined the number of misassemblies for assembled contigs from different library sizes. The rate of misassemblies is not higher for these assembled results with higher N50 (Additional file 2: Figure S1). This result suggest the higher N50 values really represent better assembly results. Because quality scores for different library sizes were similar (Additional file 1: Table S5), the advantage observed in libraries with longer sizes likely resulted from the paired-end information but not read quality. To investigate whether the higher N50 values for larger library sizes can be attributed to the paired-end information alone, we further investigated the results from Group A [SE] (Fig. 3c and d), in which the superiority of larger libraries disappeared. We noticed that the smallest library (300 bp) still had a slightly lower N50 value because most read pairs from this library have almost overlapped with each other completely. However, Group A [SE] had much worse de novo assembly results compared with Group A [PE] in all datasets, again indicating the importance of location 

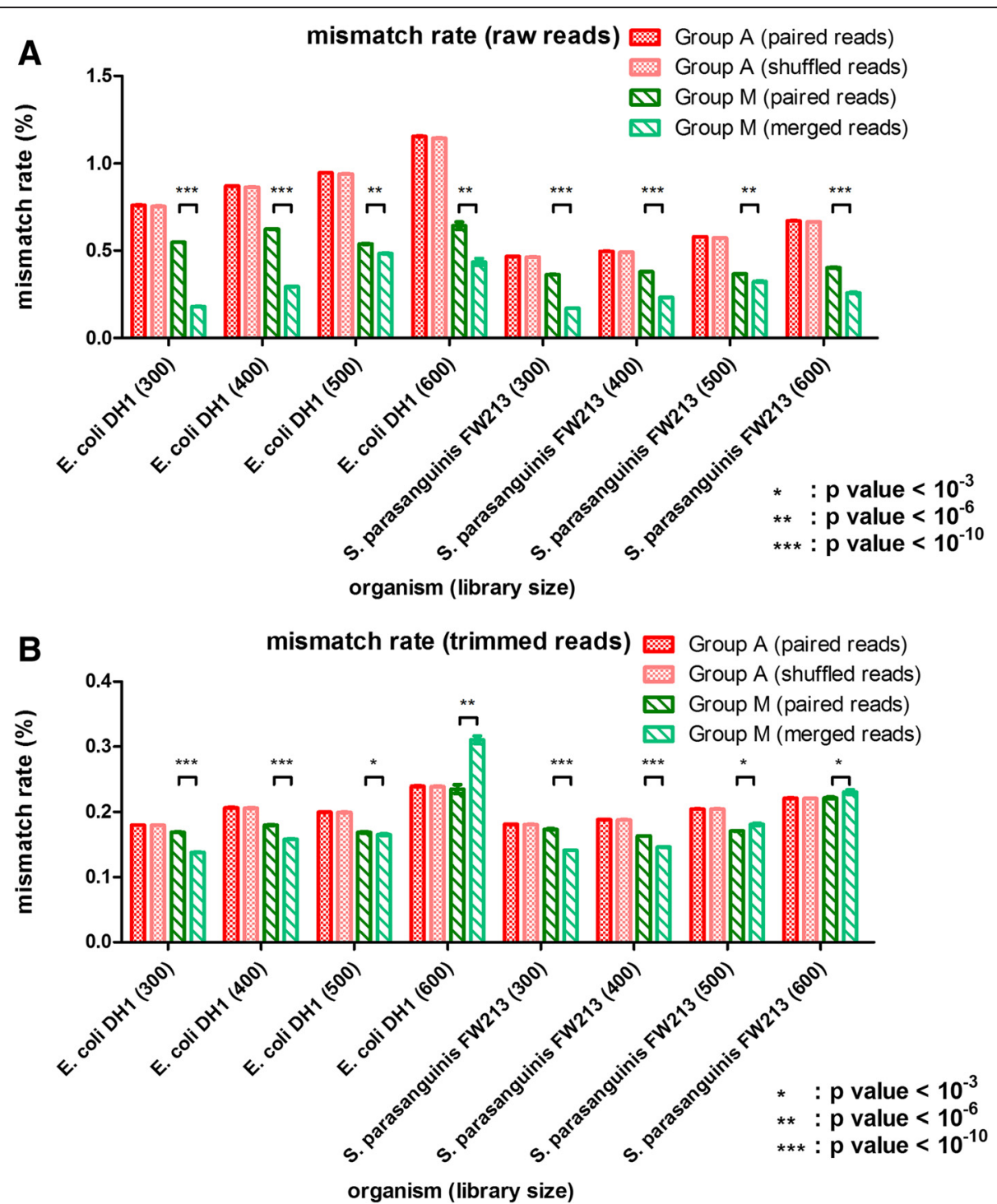

Fig. 1 Mismatch rates detected from mapping reads to reference genome. Reads from Group A (all reads) were mapped to reference genomes as paired-end reads or single end reads (R1 and R2 shuffled together). Reads from Group M (reads can be merged together) were mapped as paired-end reads or merged single reads. Mismatch rates for Group A and Group M are explored. For raw reads (a), the mismatch rates of Group A were similar for both paired-end mapping and shuffled reads mapping. The rate decreased significantly for Group M (paired-end) and decreased even more for Group M (merged reads). For trimmed reads (b), there was much less decrease or even increase of mismatch for large libraries (500 bp and 600 bp). The difference of mismatch rates between Group M (paired reads) and Group M (merged reads) was tested by paired t tests

information in the de novo assembly process. Similar patterns were found when we used different assemblers (IDBA-UD and SPAdes) as shown in Additional file 3: Figure S2 [39, 40].

\section{Merged reads improve de novo assembly only when read depth is low}

To further examine the effect of merged reads in the de novo assembly process, we focused on the subset of reads that could be merged (Group $M$ ). We assembled Group M as paired-end (Group M [PE]) or merged single end reads (Group M [SE]). The two large libraries (500 bp and $600 \mathrm{bp}$ ) were skipped in this analysis because few overlapping reads were present in these two groups. We observed that Group M [PE] and Group M [SE] had similar performances on the E. coli DH1 genome in terms of N50 and number of misassemblies (Fig. 4a and c and Additional file 4: Figure S3). However, the N50 from Group M [PE] was higher than the N50 from Group M [SE] in S. parasanguinis FW213 (Fig. 4b and d) when the depth of read coverage was higher than 20X. We suggest that this difference may be because of 


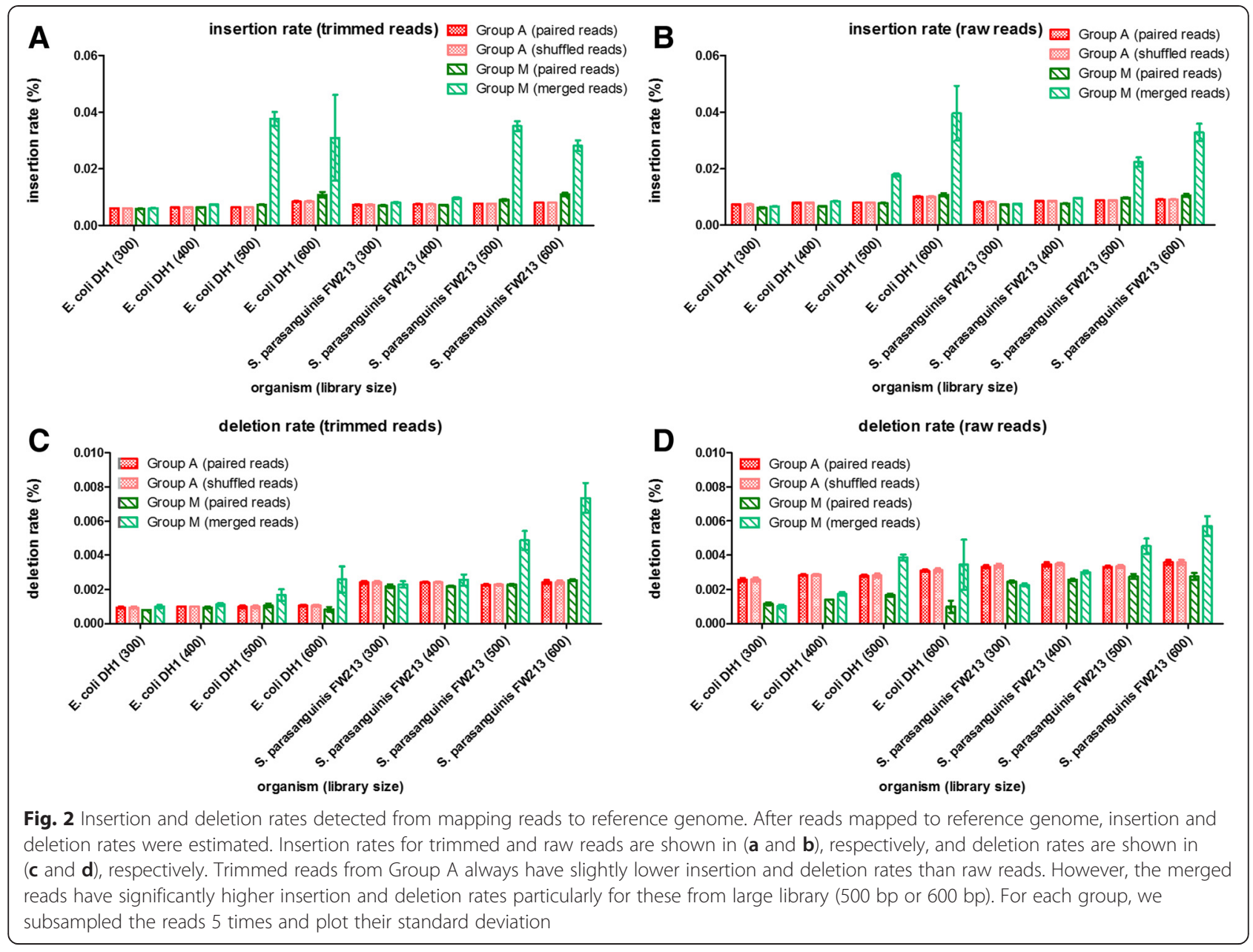

specific properties of the genome-in particular, the presence of tandem repeats that can increase the possibility of erroneously merged reads and hinder assembly. Because short tandem repeats may become sources of erroneous merges, we focused on tandem repeats shorter than 100 bps. We observed that $0.68 \%$ of the $S$. parasanguinis FW213 genome was covered by tandem repeats compared with only $0.13 \%$ of the E. coli DH1 genome. Two of the tandem repeats, $72 \mathrm{bp}$ and $36 \mathrm{bp}$, on the S. parasanguinis FW213 genome had copy numbers as high as 81 and 165, respectively. Although such high copy numbers are infrequent, S. parasanguinis FW213 has 5.4 times more tandem repeats than E. coli DH1. This higher rate of repeats also explains the higher insertion and deletion rates for S. parasanguinis FW213 in Fig. 2. The merging step may introduce errors into the middle of reads that will largely complicate the de Bruijn graph used in assembly and result in incorrect assembly results in S. parasanguinis FW213.

When the depth of read coverage was small, Group M outcompeted Group A (Fig. 4). This difference may result from the slightly higher overall quality of reads from
Group M compared with reads from Group A (Additional file 1: Table S6). Given that Group M only included reads that had overlapped regions, these reads consequently provided less location information. This result suggests that when the depth of read coverage is low, read quality is a critical factor in de novo assembly (Fig. 4). However, as the depth of read coverage increased, paired-end information could compensate and eventually provide greater benefits than higher accuracy. Similar results were observed when these reads are assembly by IDBA-UD or SPAdes (Additional file 5: Figure S4). For Group M, merged single end reads yielded an even higher N50 than the original paired-end reads (blue and light blue lines in Fig. 4). This result indicates that longer reads with higher accuracy improve the assembly results. Nevertheless, this improvement again disappears when the depth of read coverage increases. We observed that Group $M$ [SE] barely overcame Group $M[\mathrm{PE}]$ in $S$. parasanguinis FW213. We believe that this result is because of the differences in the genome complexities of E. coli DH1 and $S$. parasanguinis FW213. Although S. parasanguinis FW213 has a smaller genome, it contains 7 tandem repeats larger 


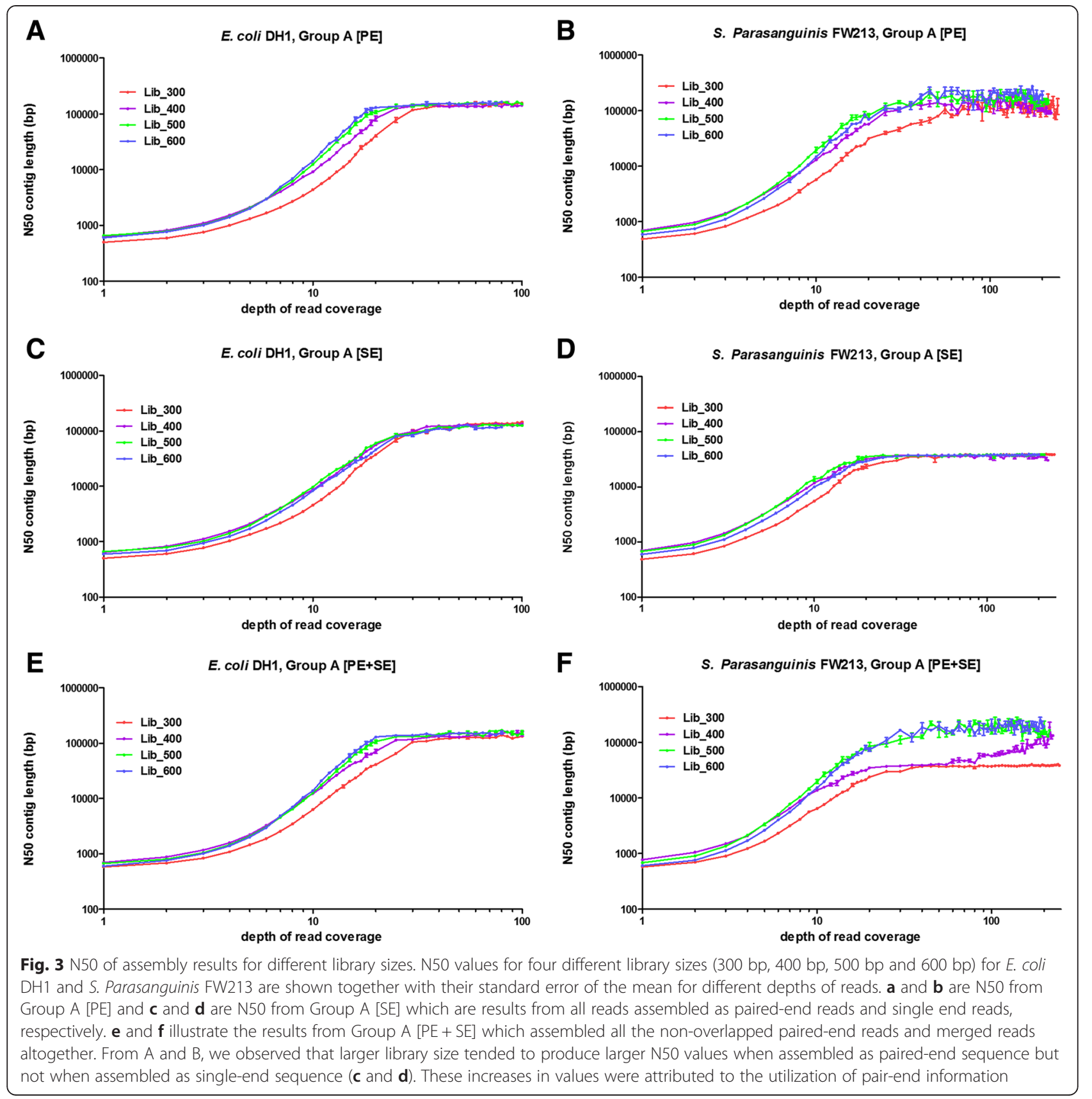

than $400 \mathrm{bp}$, and these repeats have copy numbers range from 2.2 to 6.1 (Additional file 1: Table S7). Under the same criteria, E. coli DH1 only has a repeat of 535 bp with a copy number of 2.9 .

\section{Merged reads increase indel frequency in de novo assembly when depth of read coverage is low}

Because the merging step may mistakenly introduced indels into reads, we further explored the indel rates for these assembly results. By comparing assembled contigs with reference genome we can obtain the number of indel in our assembled contigs for different assembly strategies of Group A (Fig. 5). We found that the number of indels decreases as more reads used in the assembly. This is reasonable because when depth of read coverage is low, errors in few reads may result in errors in de Bruijn graph that can be corrected when more reads are used to construct the graph. We also observed higher indel rates for large library (600 bp) when comparing between different library sizes. Although the indel rates tend to decrease as the depth of read coverage increases, there are exceptions in both $\mathrm{PE}$ and $\mathrm{PE}+\mathrm{SE}$ groups when depths of reads are low (Fig. 5). This phenomenon suggests that even if the reads were 


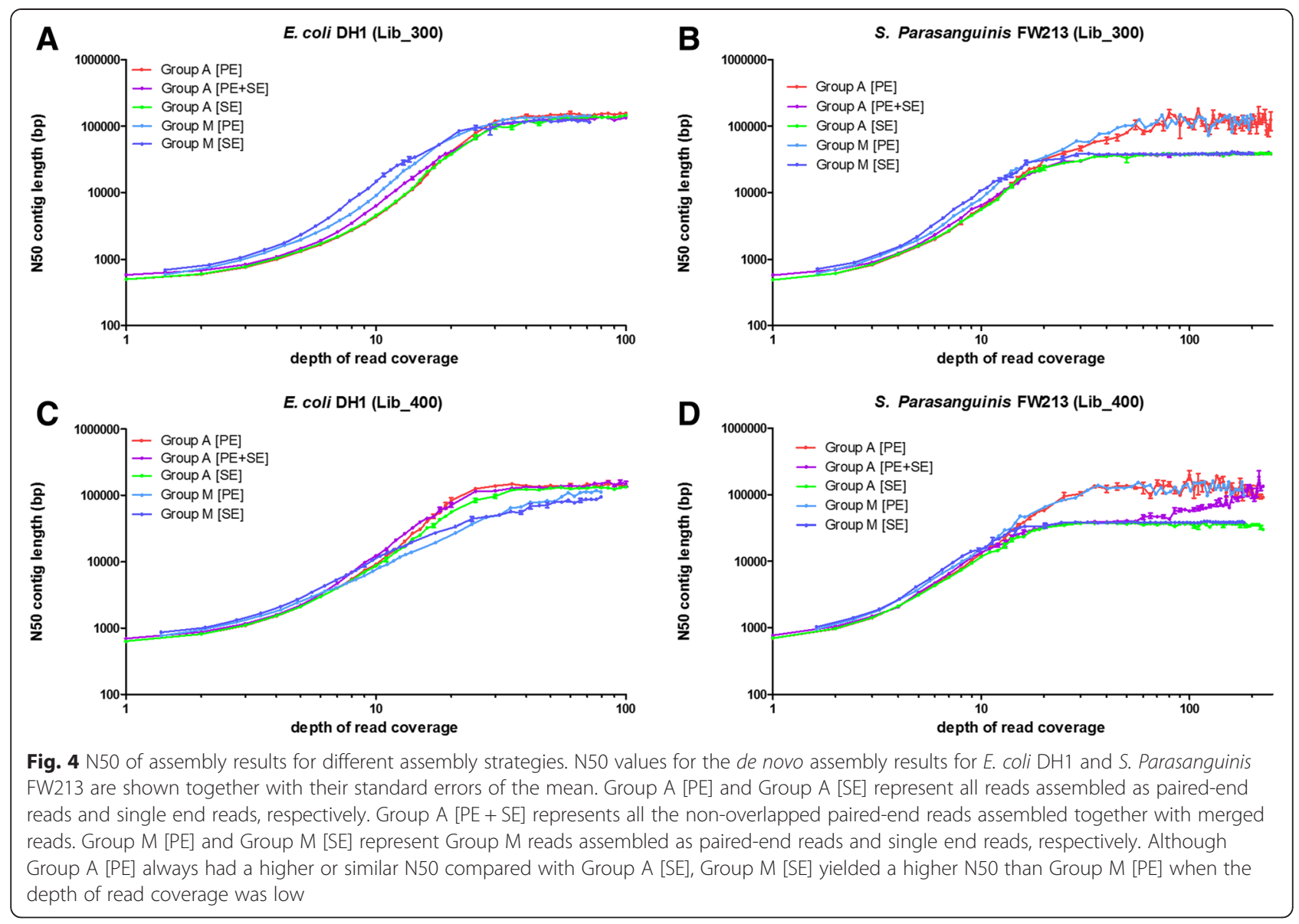

merged, the similarity in the end of read pair causes indels in the final assembly result when depths of read coverage are not high enough. It is worth mentioning that the indels rates are slightly lower in PE group comparing to $\mathrm{PE}+\mathrm{SE}$ group. This result suggests the error indels in assembled contigs are more likely to be created when paired information are used from barely overlapped paired-end reads and the merging step may augment the error rate. Nevertheless most of these indel errors can be eliminated when the read number is large enough. Even the depth of read coverage for S. parasanguinis FW213 reached more than 200X, twice the maximum depth of E. coli DH1. We observed that the number of indels in S. parasanguinis FW213 was higher than that in E. coli DH1. The higher indel rate is again consistent with the idea that the elimination of indels is more difficult for genomes containing repeats. This result demonstrates that these repeats can only be resolved by reads longer than the repeats instead of increasing depth of read coverage.

\section{Gene and genome coverage and sequencing depth}

By examining the de novo assembly results from real sequencing data, we further explored how many reads are required for de novo assembly analysis. Although the optimal sequencing depth may vary for different read length and organisms, our data can provide hints for analysis of genomes with similar characteristics. We explored how much of the genome is covered by assembled contigs. The covered genome fraction increases as sequencing depth increasing and finally reaches plateau. The genome coverage is different for different library sizes and the median library sizes (400 bp and $500 \mathrm{bp}$ ) tend to have relatively higher genome coverage fraction while depth of read coverage is low. However, as sequencing coverage depth increasing all libraries can reach to similar plateau value. We found that $97 \%$ of the genome can be covered by coverage depths approximately $12 \mathrm{X}$ 19X and 19X-50X for the larger libraries (400 bp, $500 \mathrm{bp}$ and $600 \mathrm{bp}$ ) and smallest library (300 bp), respectively (Fig. 6). We also found that the plateau value is approximately $96.2 \sim 98.2 \%$, which again supports previous results that part of the genome can never be resolved until reads larger than the long repeat regions are included in de novo assembly analysis. We also examined the covered genome fraction across different assembly strategies (Additional file 6: Figure S5). We found the group M has slightly higher coverage while the depth of read 


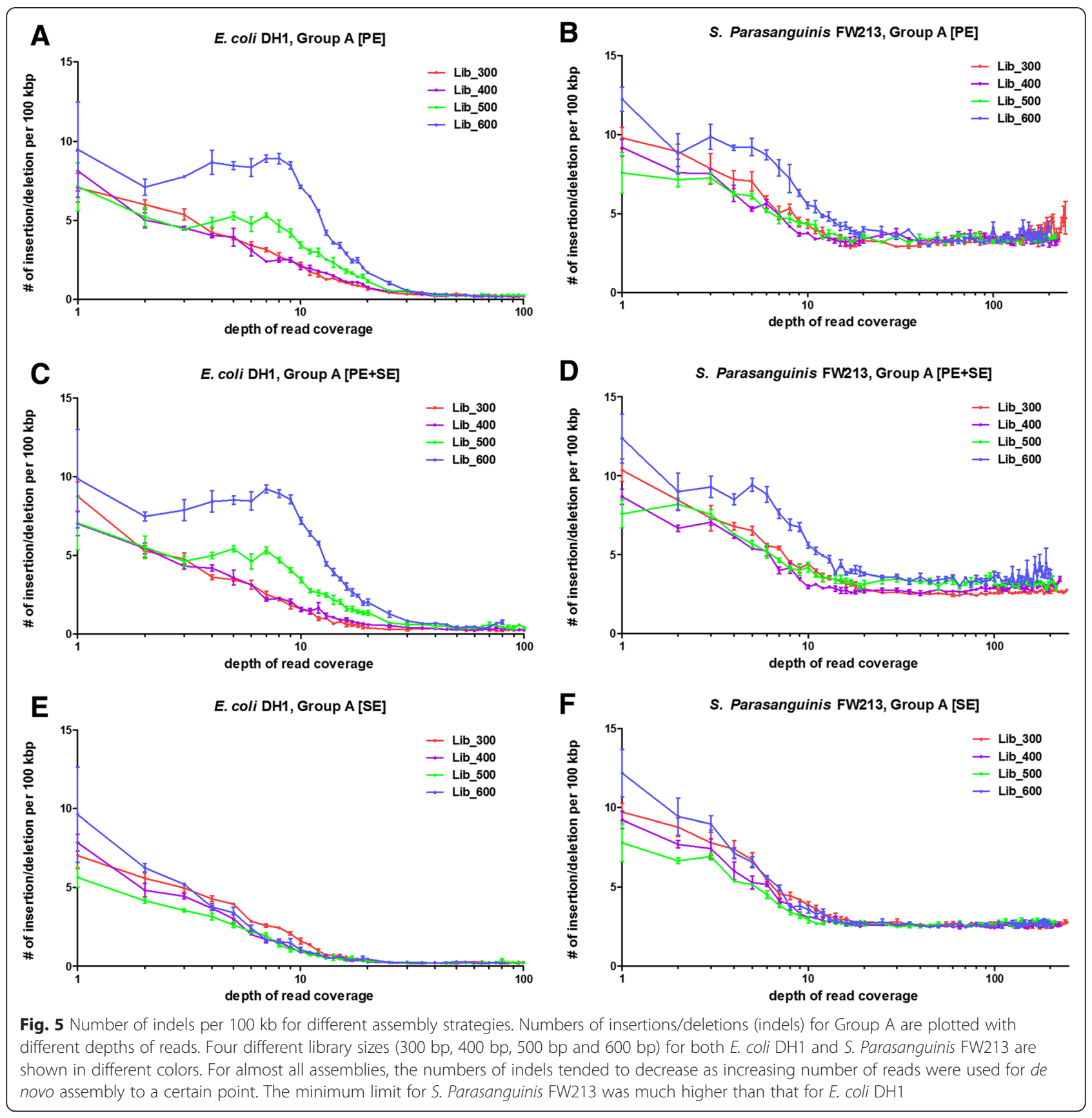

coverage is low but as depth of read coverage increasing finally all assembly strategies all reach plateau with similar covered genome fraction. We further explored how many of the annotated genes were fully covered by these assembled contigs. The pattern of gene coverage was similar to genome coverage only with relatively lower coverage that was obvious because only fully covered genes considered. The plateau value for gene coverage was $92.9 \sim 98.2 \%$.

Another suggestion for choosing read coverage depths is to generate a higher number of reads than actually necessary and then obtain a subsample from original reads. We noticed that there are fluctuations for N50 values in all assembly strategies particularly for large coverage depths (Figs. 3 and 4,). Although all of these read numbers are the same and all of the reads were already selected with their read quality and read length, different combinations of these qualified reads resulted in different assembly results. Regardless of the cause of the difference, our result suggests that when the number of reads is large enough, obtaining subsets from all of the available reads and performing multiple do novo 


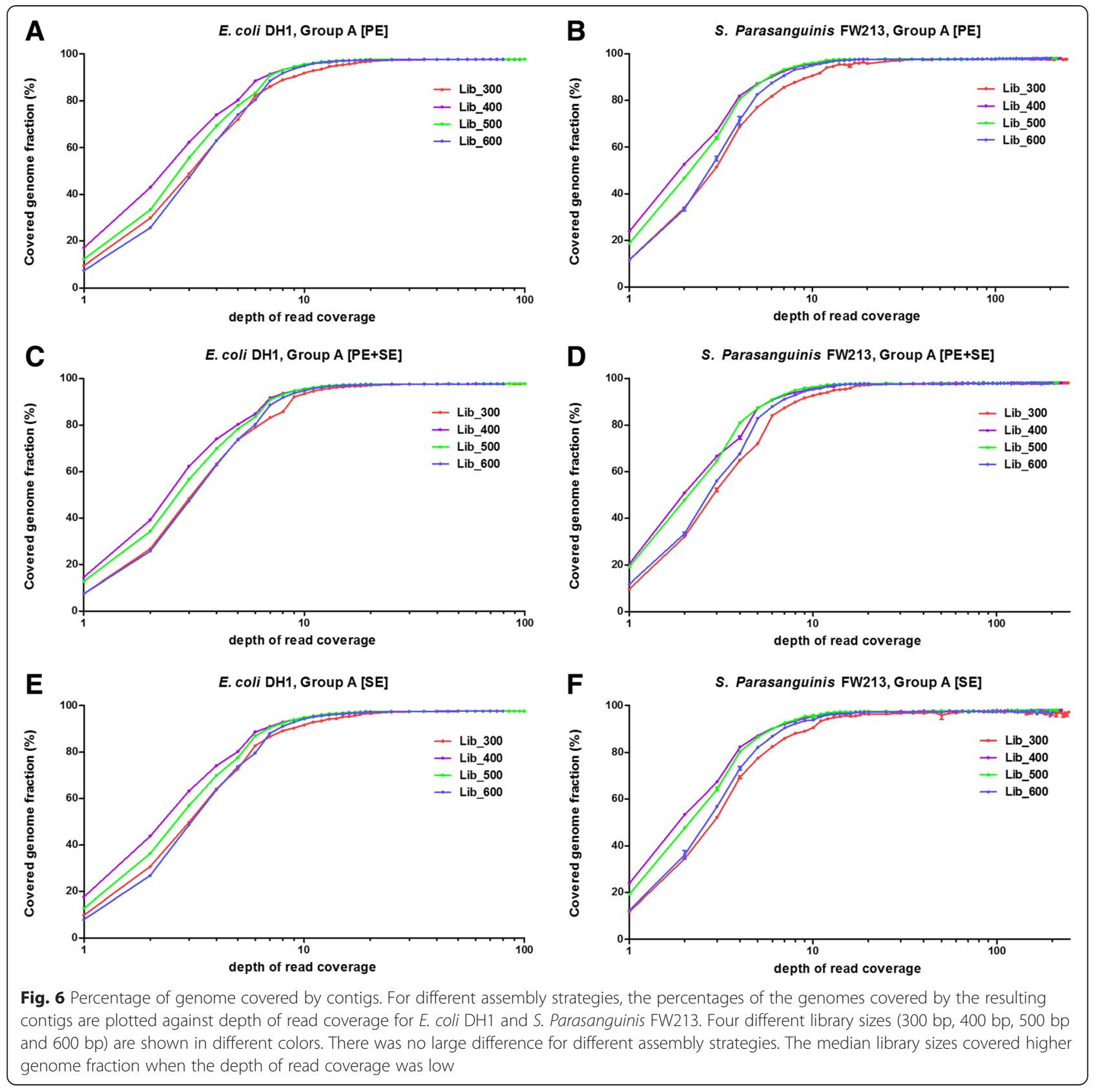

assemblies might be beneficial. From the same dataset, certain combinations of reads may provide better genome reconstruction results. The reason behind the differences in assembly results may be the sequence content of the reads, the real covered regions in the genome or other factors. Thus, further study is warranted to generate selection criteria for genome assembly studies.

\section{Conclusions}

As more and more bacteria genome reconstruction projects are conceived, it is increasingly more important to have guidelines and suggestions for designing the analyses.
In this study, we provide detailed paired-end read analyses of real sequencing data from two bacteria and generate some useful suggestions for future bacterial genome analyses. By using four different real datasets from two representative genomes, we investigated the effect of merging overlapping paired-end reads. We observed that the merging step could create longer reads with higher accuracy. We further demonstrated that the improvement in read accuracy could improve assembly when reads were fewer. However, as the read number increased, paired-end information rather than sequence length or quality is the key factor dominating the outcome of de novo assembly. In 
addition, we also observed that the merging step may unexpectedly create indels within reads that further create indels in assembled contigs. Although these indels in contigs can be eliminated when the depth of read coverage increases, the elimination is less effective for genomes with many repeats. In summary, our results suggest that merging paired-end data analysis is not always beneficial and better avoided when the genome under study contains many repeat sequences. These results shed new light on genomic sequence construction.

\section{Additional files}

Additional file 1: Table S1-S7. Table S1 - Summary of mapping reads to reference genome. Table $\mathbf{S} \mathbf{2}$ - Mismatch rates detected from reads mapped to reference genome before and after merge by different merge programs. Table $\mathbf{S} \mathbf{3}$ - Insertion rates detected from reads mapped to reference genome before and after merge by different merge programs.

Table S4 - Deletions rates detected from reads mapped to reference genome before and after merge by different merge programs.

Table S5 - Q30* values for Group A from different libraries.

Table S6 - Q30* values for Group M from different libraries.

Table S7 - Tandem repeats in S. Parasanguinis FW213. (DOCX 36 kb)

Additional file 2: Figure S1. Number of misassemblies for assembly results from different library sizes. Number of misassemblies for four different library sizes (300 bp, 400 bp, 500 bp and 600 bp) for E. coli DH1 and S. Parasanguinis FW213 are plotted against different depth of read coverage. A and B are from Group A [PE] which represents assembled results from all reads assembled as paired-end reads. $C$ and $D$ are from Group A [PE + SE] which represents assembled results from merged reads and all the non-overlapped paired end reads. E and F are from Group A [SE] which represents assembled results from all reads assembled as single-end reads. The number of misassemblies decreases as the depth of read coverage increases. Even though there are some fluctuations when depth of read coverage is low, the number of misassemblies reaches a steady number for all library sizes when depth of read coverage is high. (TIFF $882 \mathrm{~kb}$ )

Additional file 3: Figure S2. N50 for assembly results from different library sizes assembled by IDBA-UD and SPAdes. N50 values for four different library sizes (300 bp, 400 bp, 500 bp and 600 bp) for E. coli DH1 and S. Parasanguinis FW213 are plotted against different depth of read coverage. A, B, C and D are N50 from Group A [PE] and E, F, G and $H$ are N50 from Group A [SE] which are results from all reads assembled as paired-end reads and single end reads, respectively. N50 increases as the depth of read coverage increase and finally reach plateau. Different library sizes show difference in N50 values while the smallest library size (Lib_300) always gives the lowest N50 value. (TIFF $814 \mathrm{~kb}$ )

Additional file 4: Figure $\mathbf{S 3}$. Number of misassemblies for different assembly strategies. Number of misassemblies for the de novo assembly results for E. coli DH1 and S. Parasanguinis FW213 are shown together with their standard errors of the mean. Group A [PE] and Group A [SE] represent all reads assembled as paired-end reads and single end reads, respectively. Group A [PE + SE] represents all the non-overlapped pairedend reads assembled together with merged reads. Group $M[P E]$ and Group M [SE] represent Group M reads assembled as paired-end reads and single end reads, respectively. The numbers of misassemblies fluctuate a lot when depths of read number are low and gradually decreases until they reach a steady number. The paired-end reads (Group A [PE] and Group M [PE]) in S. Parasanguinis FW213 gave the lowest number of misassemblies when depths of read number are high. (TIFF $669 \mathrm{~kb}$ )

Additional file 5: Figure S4. N50 for assembly results from different assembly strategies assembled by IDBA-UD and SPAdes. N50 values for the de novo assembly results for E. coli DH1 and S. Parasanguinis FW213 by IDBA-UD and SPAdes. Group A [PE] and Group A [SE] represent all reads assembled as paired-end reads and single end reads, respectively. Group A [PE + SE] represents all the non-overlapped paired-end reads assembled together with merged reads. Group M [PE] and Group M [SE] represent Group M reads assembled as paired-end reads and single end reads, respectively. Group M slightly outcompete Group A when depth of read coverage is low. However, Group A always has the highest N50 values when depth of read coverage is high. (TIFF $867 \mathrm{~kb}$ )

Additional file 6: Figure S5. Percentage of genome covered by contigs assembled by different assembly strategies. Covered genome fraction are shown for library size $300 \mathrm{bp}$ and $400 \mathrm{bp}$. Group A includes all reads without selection and Group $M$ are read that containing overlapped region. The covered fraction increases as depth of read coverage increases. Higher percentage were found for Group M comparing to Group A when depth of read coverage are low. However, all strategies reach similar percentage of genome coverage when depths of read coverage are high. (TIFF $457 \mathrm{~kb}$ )

\section{Competing interests}

The authors declare that they have no competing interests.

\section{Authors' contributions}

TWC designed the experiment and wrote the manuscript. CYL and RCG performed the experiment. TWC, YFC and THW analyzed the data. WCL, CCL and PJH provided the computing system. YYC and CHC provided DNA samples and revised the manuscript. PT supported this study and revised the manuscript. All authors read and approved the manuscript.

\section{Acknowledgements}

This work is supported by the Ministry of Science and Technology (MOST-1032319B-400-001) CMRPD 1A0582 from Chang Gung Memorial Hospital to PT.

\section{Author details}

'Bioinformatics Core Laboratory, Molecular Medicine Research Center, Chang Gung University, Taoyuan, Taiwan. ${ }^{2}$ Institute of Biomedical Informatics, National Yang-Ming University, Taipei, Taiwan. ${ }^{3}$ Sequencing Technology Ltd, Taipei, Taiwan. ${ }^{4}$ Department of Microbiology and Immunology, Chang Gung University, Taoyuan, Taiwan. ${ }^{5}$ Graduate Institute of Biomedical Sciences, Chang Gung University, Taoyuan, Taiwan. ' ${ }^{6}$ Molecular Infectious Diseases Research Center, Chang Gung Memorial Hospital, Taoyuan, Taiwan.

Received: 4 March 2015 Accepted: 18 August 2015

Published online: 28 August 2015

\section{References}

1. Shendure J, Ji H. Next-generation DNA sequencing. Nat Biotechnol. 2008;26(10):1135-45.

2. Mikheyev AS, Tin MM. A first look at the Oxford Nanopore MinION sequencer. Mol Ecol Resour. 2014;14(6):1097-102.

3. Koren S, Phillippy AM. One chromosome, one contig: complete microbial genomes from long-read sequencing and assembly. Curr Opin Microbiol. 2014;23C:110-20.

4. Goodwin SS, Gurtowski J, Ethe-Sayers S, Deshpande P, Schatz M, McCombie R. Oxford Nanopore Sequencing and de novo Assembly of a Eukaryotic Genome. BioRxiv pre-print server. 2015.

5. Koren S, Schatz MC, Walenz BP, Martin J, Howard JT, Ganapathy G, et al. Hybrid error correction and de novo assembly of single-molecule sequencing reads. Nat Biotechnol. 2012;30(7):693-700.

6. Chin CS, Alexander DH, Marks P, Klammer AA, Drake J, Heiner C, et al. Nonhybrid, finished microbial genome assemblies from long-read SMRT sequencing data. Nat Methods. 2013;10:563-9.

7. McCoy RC, Taylor RW, Blauwkamp TA, Kelley JL, Kertesz M, Pushkarev D, et al. Illumina TruSeq synthetic long-reads empower de novo assembly and resolve complex, highly-repetitive transposable elements. PLoS One. 2014;9(9):e106689.

8. Luo R, Liu B, Xie Y, Li Z, Huang W, Yuan J, et al. SOAPdenovo2: an empirically improved memory-efficient short-read de novo assembler. Gigascience. 2012;1 (1):18. 
9. Li R, Zhu H, Ruan J, Qian W, Fang X, Shi Z, et al. De novo assembly of human genomes with massively parallel short read sequencing. Genome Res. 2010;20(2):265-72.

10. Reinhardt JA, Baltrus DA, Nishimura MT, Jeck WR, Jones CD, Dangl JL. De novo assembly using low-coverage short read sequence data from the rice pathogen Pseudomonas syringae pv. oryzae. Genome Res. 2009;19(2):294-305.

11. Zerbino DR, Birney E. Velvet: algorithms for de novo short read assembly using de Bruijn graphs. Genome Res. 2008;18(5):821-9.

12. Hernandez D, Francois $P$, Farinelli L, Osteras M, Schrenzel J. De novo bacterial genome sequencing: millions of very short reads assembled on a desktop computer. Genome Res. 2008;18(5):802-9.

13. Chaisson MJ, Pevzner PA. Short read fragment assembly of bacterial genomes. Genome Res. 2008;18(2):324-30.

14. Butler J, MacCallum I, Kleber M, Shlyakhter IA, Belmonte MK, Lander ES, et al. ALLPATHS: de novo assembly of whole-genome shotgun microreads. Genome Res. 2008;18(5):810-20.

15. Chaisson MJ, Brinza D, Pevzner PA. De novo fragment assembly with short mate-paired reads: Does the read length matter? Genome Res. 2009;19(2):336-46.

16. Whiteford N, Haslam N, Weber G, Prugel-Bennett A, Essex JW, Roach PL, et al. An analysis of the feasibility of short read sequencing. Nucleic Acids Res. 2005;33(19):e171.

17. Pevzner PA, Tang H, Waterman MS. An Eulerian path approach to DNA fragment assembly. Proc Natl Acad Sci U S A. 2001;98(17):9748-53.

18. Zhang W, Chen J, Yang Y, Tang Y, Shang J, Shen B. A practical comparison of de novo genome assembly software tools for next-generation sequencing technologies. PLoS One. 2011;6(3):e17915.

19. Haiminen N, Kuhn DN, Parida L, Rigoutsos I. Evaluation of methods for de novo genome assembly from high-throughput sequencing reads reveals dependencies that affect the quality of the results. PLoS One. 2011;6(9):e24182

20. Kelley DR, Schatz MC, Salzberg SL. Quake: quality-aware detection and correction of sequencing errors. Genome Biol. 2010;11(11):R116.

21. Tammi MT, Arner E, Kindlund E, Andersson B. Correcting errors in shotgun sequences. Nucleic Acids Res. 2003;31(15):4663-72.

22. Zhang J, Kobert K, Flouri T, Stamatakis A. PEAR: a fast and accurate Illumina Paired-End reAd mergeR. Bioinformatics. 2014;30(5):614-20.

23. FLASH: Fast Length Adjustment of Short Reads to Improve Genome Assemblies. [http://www.cbcb.umd.edu/software/flash]

24. ea-utils: Command-line tools for processing biological sequencing data. [http://code.google.com/p/ea-utils]

25. Stitch. [https://github.com/audy/stitch]

26. CLC Genomics Workbench 7.0.4. [http://www.clcbio.com]

27. Masella AP, Bartram AK, Truszkowski JM, Brown DG, Neufeld JD. PANDAseq: paired-end assembler for illumina sequences. BMC Bioinformatics. 2012;13:31.

28. Loman NJ, Misra RV, Dallman TJ, Constantinidou C, Gharbia SE, Wain J, et al. Performance comparison of benchtop high-throughput sequencing platforms. Nat Biotechnol. 2012;30(5):434-9.

29. Sims D, Sudbery I, llott NE, Heger A, Ponting CP. Sequencing depth and coverage: key considerations in genomic analyses. Nat Rev Genet. 2014;15(2):121-32.

30. Desai A, Marwah VS, Yadav A, Jha V, Dhaygude K, Bangar U, et al. Identification of optimum sequencing depth especially for de novo genome assembly of small genomes using next generation sequencing data. PLoS One. 2013;8(4):e60204.

31. Chen YY, Clancy KA, Burne RA. Streptococcus salivarius urease: genetic and biochemical characterization and expression in a dental plaque streptococcus. Infect Immun. 1996;64(2):585-92.

32. seq_crumbs. [http://bioinf.comav.upv.es/seq_crumbs/]

33. Tatusova T, Ciufo S, Fedorov B, O'Neill K, Tolstoy I. RefSeq microbial genomes database: new representation and annotation strategy. Nucleic Acids Res. 2014;42(Database issue):D553-9.

34. seqtk. [https://github.com/lh3/seqtk]

35. Li H, Durbin R. Fast and accurate short read alignment with Burrows-Wheeler transform. Bioinformatics. 2009;25(14):1754-60.

36. Benson G. Tandem repeats finder: a program to analyze DNA sequences. Nucleic Acids Res. 1999;27(2):573-80.
37. Gurevich A, Saveliev V, Vyahhi N, Tesler G. QUAST: quality assessment tool for genome assemblies. Bioinformatics. 2013;29(8):1072-5.

38. Miller JR, Koren S, Sutton G. Assembly algorithms for next-generation sequencing data. Genomics. 2010;95(6):315-27.

39. Peng Y, Leung HC, Yiu SM, Chin FY. IDBA-UD: a de novo assembler for single-cell and metagenomic sequencing data with highly uneven depth. Bioinformatics. 2012;28(11):1420-8.

40. Bankevich A, Nurk S, Antipov D, Gurevich AA, Dvorkin M, Kulikov AS, et al. SPAdes: a new genome assembly algorithm and its applications to singlecell sequencing. J Comput Biol. 2012;19(5):455-77.

\section{Submit your next manuscript to BioMed Central and take full advantage of:}

- Convenient online submission

- Thorough peer review

- No space constraints or color figure charges

- Immediate publication on acceptance

- Inclusion in PubMed, CAS, Scopus and Google Scholar

- Research which is freely available for redistribution 